\title{
Effect of Varying Displays and Room Illuminance on Caries Diagnostic Accuracy in Digital Dental Radiographs
}

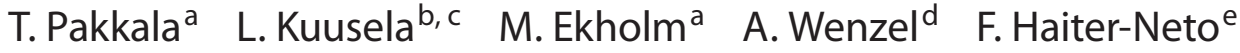 \\ M. Kortesniemi ${ }^{\text {b, } c}$

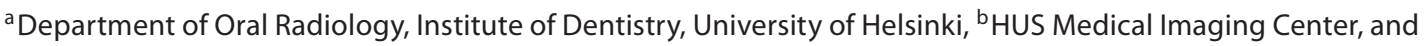 \\ 'Department of Physics, University of Helsinki, Finland; d Department of Oral Radiology, School of Dentistry, Aarhus \\ University, Aarhus, Denmark; ${ }^{e}$ Department of Oral Diagnosis, Piracicaba Dental School, State University of Campinas, \\ Piracicaba, Brazil
}

\section{Key Words}

Caries diagnostics $\cdot$ Digital radiography $\cdot$ Display $\cdot$

Illuminance

\begin{abstract}
In clinical practice, digital radiographs taken for caries diagnostics are viewed on varying types of displays and usually in relatively high ambient lighting (room illuminance) conditions. Our purpose was to assess the effect of room illuminance and varying display types on caries diagnostic accuracy in digital dental radiographs. Previous studies have shown that the diagnostic accuracy of caries detection is significantly better in reduced lighting conditions. Our hypothesis was that higher display luminance could compensate for this in higher ambient lighting conditions. Extracted human teeth with approximal surfaces clinically ranging from sound to demineralized were radiographed and evaluated by 3 observers who detected carious lesions on 3 different types of displays in 3 different room illuminance settings ranging from low illumination, i.e. what is recommended for diagnostic viewing, to higher illumination levels corresponding to those found in an average dental office. Sectioning and microscopy of the teeth validated the presence or absence
\end{abstract}

of a carious lesion. Sensitivity, specificity and accuracy were calculated for each modality and observer. Differences were estimated by analyzing the binary data assuming the added effects of observer and modality in a generalized linear model. The observers obtained higher sensitivities in lower illuminance settings than in higher illuminance settings. However, this was related to a reduction in specificity, which meant that there was no significant difference in overall accuracy. Contrary to our hypothesis, there were no significant differences between the accuracy of different display types. Therefore, different displays and room illuminance levels did not affect the overall accuracy of radiographic caries detection.

Copyright $\odot 2012$ S. Karger AG, Basel

Digital radiographic images are viewed on a computer display. Patient records are currently also stored in digital form, and in the dental office the same computer and display are often used both for maintaining patient records and for viewing radiographs. The interpretation of radiographic images may, however, place other requirements on the display and viewing conditions than do the maintenance of patient records and related activities.

\section{KARGER \\ Fax +4161306 1234 \\ E-Mail karger@karger.ch}

www.karger.com
(C) 2012 S. Karger AG, Basel

$0008-6568 / 12 / 0466-0568 \$ 38.00 / 0$

Accessible online at:

www.karger.com/cre
Tuomas Pakkala

Department of Oral Radiology, Institute of Dentistry

PL 41 (Mannerheimintie 172)

FI-00014 Helsingin yliopisto (Finland)

E-Mail tuomas.pakkala@ helsinki.fi 
The ability of the display to adequately present lowcontrast structures is vital for diagnostic radiology. The properties of displays used to view radiographs in clinical practice vary widely from off-the-shelf colour displays to high-resolution Digital Image and Communication in Medicine (DICOM) Grayscale Standard Display Function (GSDF) calibrated medical displays [NEMA, 2008]. The display's bit depth, resolution and particularly the luminance and contrast response calibration may influence the quality of the diagnostic information that can be extracted from the radiograph [Samei et al., 2005]. It has been established that the luminance of the display in conjunction with the room illuminance affect the reliability of caries lesion detection [Hellén-Halme et al., 2008].

The national guidelines regarding grayscale calibration and acceptable illuminance levels in the room used for radiographic interpretation are based on guidelines of the American Association of Physicists in Medicine (AAPM) [Samei et al., 2005] and the Institute of Physics and Engineering in Medicine (IPEM) [IPEM, 2005]. These conditions are rarely met in a dentist's clinical practice, where much higher room illuminance is usually recommended for clinical work. The aim of this study was to assess the effect of varying display types and room illuminance on caries diagnostic accuracy, and to establish whether a minimum luminance setting for medical displays in high room illuminance can be recommended. We hypothesize that higher display luminance could compensate for high room illuminance in a dental office environment, where dimming the lights during clinical work is not always feasible.

\section{Materials and Methods}

Three types of display were tested in three different levels of ambient lighting. We define room illuminance as the level of lighting in a room supplied by diverse sources, e.g. sunlight from windows and fluorescent lights, and it is measured in a predetermined location directly in front of or on the surface of a display. Display luminance is the capacity of the display to project light as measured from its surface; the luminance ratio reflects the difference between the maximum light that a display actually emits as opposed to the minimum light it can emit when it is switched on, including the ambient luminance. In the case of displays with the same luminance ratio and calibration, the contrast is perceived similarly [Samei et al., 2005]. Ambient luminance is the amount of light reflected from the display surface measured from that surface when the display is switched off. The higher the illuminance in a given space, the higher the ambient luminance from the surface of the monitor, and the greater the negative effect on monitor luminance ratio.

The displays chosen for the experiment were an off-the-shelf colour display, of a type which might be found at a dental practice
(Dell 2007FP, Dell Inc., Round, Rock, Tex., USA), a DICOM-calibrated colour liquid crystal display (LCD) clinical viewing station (Eizo Flexscan MX210, Eizo Corp., Ishikawa, Japan) and a DICOM-calibrated grayscale LCD (Barco MDCG2121CB, Barco N.V., Kortrijk, Belgium) display designed for medical diagnostic work.

The quality of the displays was assessed at $8 \pm 1$ lux, using the following test images: the AAPM TG-18 QC was used for general visual image quality assessment (luminance and contrast; resolution and artefacts), AD for diffuse reflection, UN10/UN80 for luminance non-uniformity and LN1-LN18 test patterns to measure luminance and contrast response. The mean and maximum deviation to the GSDF standard was calculated for the luminance and contrast response. The luminance ratio and luminance nonuniformity were calculated as in the 2nd AAPM TG18 report:

$[200 \cdot($ L_max - L_min $) /($ L_max + L_min $)]$, where L_max and L_min are the measured maximum and minimum luminances from test patterns (UN10 and UN80).

The luminance and contrast responses were found to be those recommended for the Barco display. The contrast response for the Eizo display was not according to recommendation for low luminance values. This might be due to a non-optimal ambient luminance setting in the calibration software, i.e. the ambient luminance level had originally been set higher than what was measured on the surface of the display. As mentioned earlier, ambient luminance is caused by room illuminance and describes the light flux from the display due to reflection. It can, therefore, reduce the luminance ratio. The Dell display has extremely high luminance non-uniformity (luminance deviation for the central and peripheral parts of the display). So in this study, the actual calibration of the displays used corresponds to current clinical practice rather than an ideal situation with optimal calibration of the displays. For all other assessed quality properties, no significant differences were observed between displays. These results are represented in table 1.

The displays were tested at three room illuminance levels, measured on the display surface and on the table directly in front of it when it was on standby. The display surface was chosen as the reference location for this study. The values measured on the table surface in front of the display are included as supplementary data and to help with cross-referencing to the literature. Illuminance and luminance values were measured using an Iba Docimetry LXCan (Iba dosimetry, Schwarzenbruck, Germany).

The room illuminance levels chosen for this study were: (1) the maximum illuminance in a dental office (approx. 400 lux), (2) $50 \%$ of the maximum illuminance (approx. $200 \mathrm{lux}$ ) and (3) the recommended 15 lux as measured on the surface of the display. The dental office room illuminance had been measured earlier on the table and display surface. The measurements were taken only in one office, but were believed to be adequate; a previous study [Hellén-Halme et al., 2007] reported similar settings (a mean illuminance of 668 lux measured on the table in front of the display in a sample of 19 clinics). To ensure the same viewing conditions, the assessment was always performed at the same location, with the room illuminance level being confirmed each time and adjusted if necessary. The illuminance measured on the surface of the display and on the table top is presented in table 2 .

One hundred extracted human premolars and molars were used for the experiment. None had approximal lesion cavitation, but were either clinically sound or showed various degrees of de- 
mineralisation ranging from chalky-white to brownish discoloration. The teeth were examined with a storage phosphor plate system (Digora FMX, Soredex, Helsinki, Finland) as described previously [Isidor et al., 2009]. They were mounted in 20 silicone blocks of 5 teeth each: 1 canine (non-test tooth), 2 premolars and 2 molars. They were placed in an anatomical position with approximal surfaces in contact. The blocks were placed in a jig to provide a central beam orientation, and each tooth was radiographed individually under standard conditions $(65 \mathrm{kVp}, 10 \mathrm{~mA}$, paralleling technique, rectangular collimation with a 20 -millimetre toothreceptor distance and a 320-millimetre focus-to-receptor distance) with an intraoral X-ray unit (Gendex 1000 DC X-ray unit, Gendex, Des Moines, Ill., USA). The images were saved in taggedimage file format and imported into a general program (UniscoreL, Erik Gotfredsen, School of Dentistry, Aarhus University, Denmark). This program has image-enhancement facilities to allow for adjustment of contrast, brightness, $\gamma$-curve function and magnification. The quality of the radiographic images acquired was comparable to those produced in vivo in a clinical setting.

Two dentists in their first year of specialist training in oral radiology and a specialist evaluated the images on a lesion depth scale: $0=$ no lesion, $1=$ a carious lesion extending no further than half the depth of the enamel, 2 = a carious lesion extending deeper than half the depth of the enamel and $3=$ a carious lesion extending into the dentine. At first, a training session was held to allow the observers to get acquainted with the program. A set of images was then evaluated by all observers in a given room-illuminance setting on each of the displays in turn. The room illuminance was then changed and the procedure was repeated. The evaluation sequence of display and room illuminance was randomized. The sequence of images displayed within a given setting was also randomized. The evaluations were spread out over a number of days in order to avoid the effects of fatigue and recall bias.

After scoring the images, microscopy of tooth sections served as a validation for the presence or absence of a lesion. The teeth were embedded in acrylic (Vipcril, Vipi, São Paulo, Brazil) and sectioned into $700-\mu \mathrm{m}$-thick sections with a low-speed diamond saw (Nheel Exakt, South Bay Technology, Calif., USA). The sections were then glued on microscope glasses and evaluated by 2 experienced observers. A carious lesion was judged to be present when an opaque white-to-brown discoloration was found to be present in an area at risk for caries. If a lesion was discovered on a surface, the section showing the largest extent of the lesion was accepted as the 'gold standard' for that surface. In the microscopic analysis, 101 surfaces were found to be intact and 59 had a carious lesion. The lesions were mostly small, with $94 \%$ of them in the enamel and only $6 \%$ extending less than one third of the thickness of the dentin.

For each observer and each radiographic modality, the sensitivity, specificity and overall accuracy (true positives + true negatives or all scores) were computed by comparing the radiographic scores to the histological examination. The difference in sensitivity, specificity and accuracy was estimated by analyzing the binary data assuming the added effects of observer and modality in a generalized linear model using an identity link. The correlation within surfaces was adjusted for by applying robust standard errors. Pairwise comparisons between modalities were performed by Wald tests. The statistical analyses were made in Stata (Stata 10.1, StataCorp, College Station, Tex., USA) and the level of statistical significance was $\mathrm{p}<0.05$.
Table 1. Results from the display quality assessment

\begin{tabular}{lrrr}
\hline & Dell & Eizo & Barco \\
\hline Maximum luminance, cd/m ${ }^{2}$ & 163 & 165 & 513 \\
Luminance ratio R & 510 & 1,105 & 641 \\
Non-uniformity (UN-10), \% & 64 & 25 & 16 \\
Non-uniformity (UN-80), \% & 6 & 6 & 3 \\
Luminance response (mean error), \% & 3 & 1 & 0 \\
Luminance response (maximum error), \% & 24 & 9 & 0 \\
Contrast response (mean error), \% & 2 & 4 & 1 \\
Contrast response (maximum error), \% & 18 & 35 & 5 \\
\hline
\end{tabular}

Table 2. Room illuminance for carious lesion assessment

\begin{tabular}{lll}
\hline & $\begin{array}{l}\text { Display } \\
\text { surface, lux }\end{array}$ & $\begin{array}{l}\text { Table } \\
\text { surface, lux }\end{array}$ \\
\hline Dentist office & 414 & 709 \\
1. Maximum illuminance & $423 \pm 13$ & $763 \pm 11$ \\
2. Half of maximum illuminance & $206 \pm 2$ & $423 \pm 13$ \\
3. Minimum illuminance & $15 \pm 1$ & $30 \pm 0$ \\
\hline
\end{tabular}

Display surface was the reference location for illuminance measurements in this study. Table surface values were measured for complementary data and as a cross-reference to literature.

\section{Results}

Mean sensitivity, specificity and accuracy for the 3 displays at different room illuminance levels are presented in table 3. Mean sensitivity was in the range of $16-25 \%$. Mean specificity was in the range of $89-95 \%$. Sensitivity was statistically significantly better in the lower illuminance of 15 lux as expected, with little or no difference in scores between 200 and 400 lux. The mean sensitivity was slightly higher for the Barco display than both the Dell and Eizo displays, especially at the low illuminance level. As the room illuminance increased, however, these differences as well as overall sensitivity decreased. Surprisingly, however, lower room illuminance seemed to result in lower specificity, meaning more false-positive scores. Specificity seemed to improve as room illuminance increased from 15 to 200 lux and improved statistically significantly as it increased finally to 400 lux. At lower illuminance settings, the mean specificity for the Barco display was slightly lower than for the others. There seemed to be no significant difference in overall accuracy between observations made at different room illuminance 
Table 3. Sensitivity, specificity and accuracy (mean between observers) for displays and room illuminance levels (lux in front of the display)

\begin{tabular}{|c|c|c|c|c|c|c|c|c|c|c|}
\hline \multirow[t]{2}{*}{ Display } & \multirow[t]{2}{*}{ Illuminance } & \multicolumn{3}{|c|}{ Sensitivity, \% } & \multicolumn{3}{|c|}{ Specificity, \% } & \multicolumn{3}{|c|}{ Accuracy, \% } \\
\hline & & mean & $\min$. & $\max$ & mean & $\min$. & $\max$ & mean & $\min$. & $\max$. \\
\hline Barco & 15 & 25 & 19 & 30 & 89 & 84 & 94 & 66 & 63 & 70 \\
\hline Eizo & 15 & 21 & 20 & 24 & 92 & 92 & 93 & 66 & 66 & 67 \\
\hline Dell & 15 & 21 & 20 & 24 & 92 & 92 & 93 & 66 & 66 & 67 \\
\hline Barco & 200 & 20 & 16 & 23 & 90 & 83 & 96 & 64 & 61 & 68 \\
\hline Eizo & 200 & 16 & 7 & 22 & 93 & 89 & 95 & 64 & 62 & 65 \\
\hline Dell & 200 & 17 & 11 & 28 & 95 & 90 & 93 & 66 & 65 & 67 \\
\hline Barco & 400 & 19 & 15 & 26 & 95 & 90 & 98 & 67 & 66 & 67 \\
\hline Eizo & 400 & 17 & 11 & 25 & 94 & 89 & 97 & 66 & 65 & 66 \\
\hline Dell & 400 & 16 & 13 & 20 & 95 & 92 & 98 & 66 & 64 & 70 \\
\hline
\end{tabular}

Table 4. Comparison of multiple variables: concerning studied displays, illuminances and observers

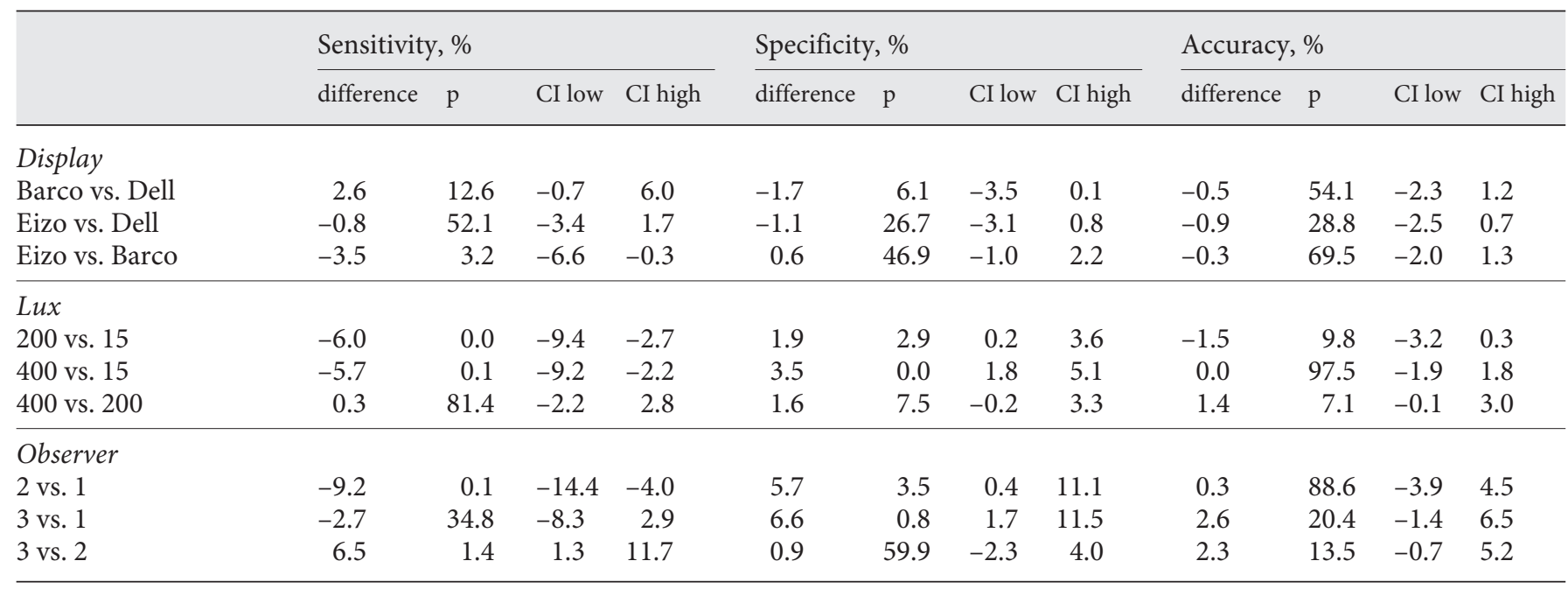

levels, and the differences between displays were small and not statistically significant anyway.

Table 4 presents a statistical comparison between displays, room illuminance levels and observers. For the overall accuracy, the differences were not statistically significant. Neither display type nor room illuminance had a significant effect on the overall accuracy of caries lesion detection.

Figures 1-3 present the sensitivity, specificity and accuracy for the 3 observers and the mean value over observers for the various illuminance levels and displays. The observers scored rather differently, but the only statistically significant difference was in sensitivity between the specialist and 1 of the dentists.

Effect of Displays and Room Lighting on Caries Diagnostics

\section{Discussion}

The carious lesions included in the study were, for the main part, shallow and restricted to the enamel. While the stage of lesion cavitation is a relevant threshold for invasive treatment, the purpose of this study was not to differentiate between lesions needing restorative treatment versus those needing increased prophylactic measures. Rather, the aim was related to the accuracy of diagnosing low-contrast differences on various grades of monitors and in various lighting conditions, for which purpose relatively small carious lesions were considered optimal. Carious lesions restricted to the enamel normally do not necessitate restorative treatment, but they may 


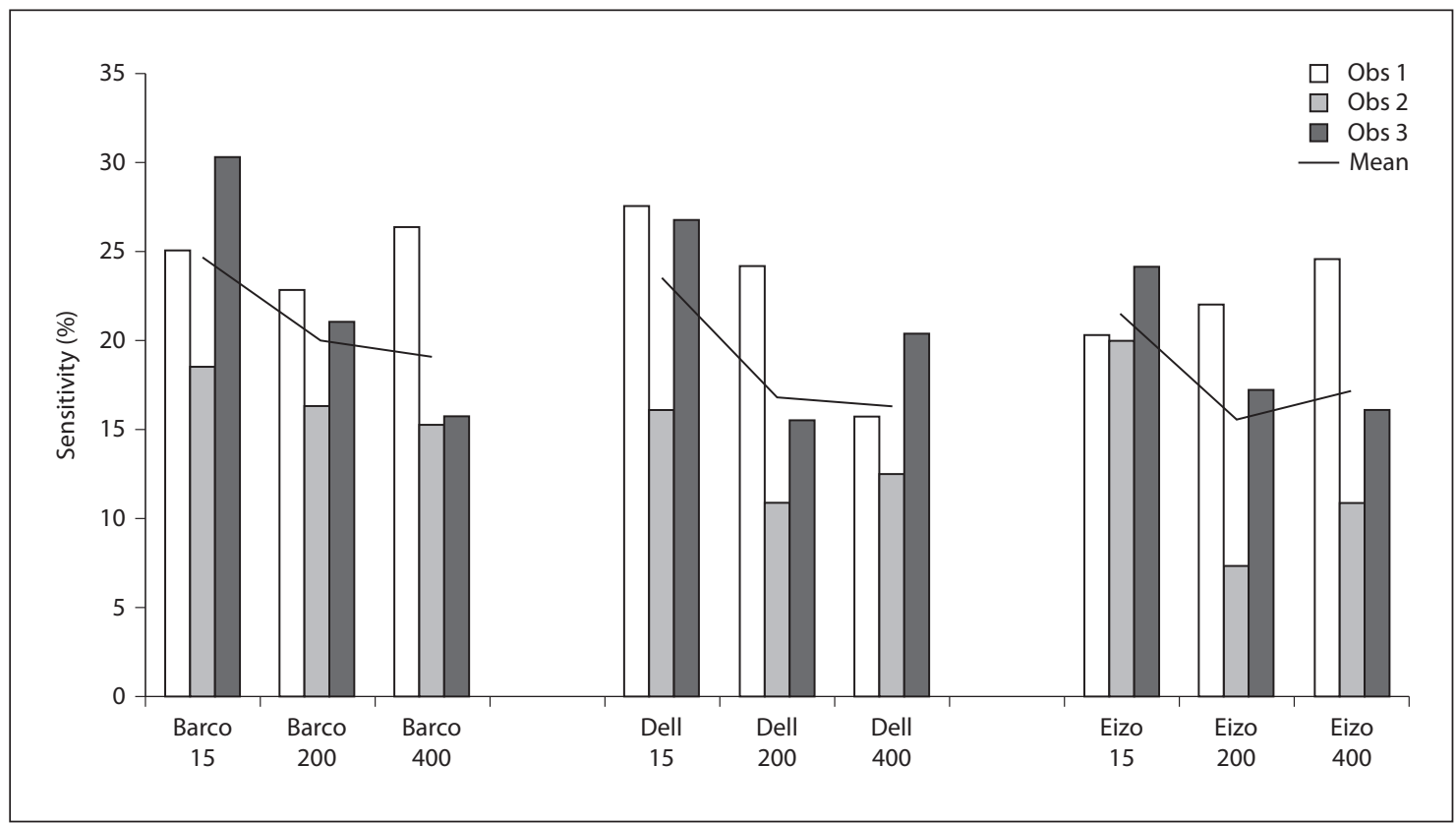

Fig. 1. Sensitivity for the 3 observers and the mean value (black line) for the room illuminance levels (lux on the display surface) and displays.

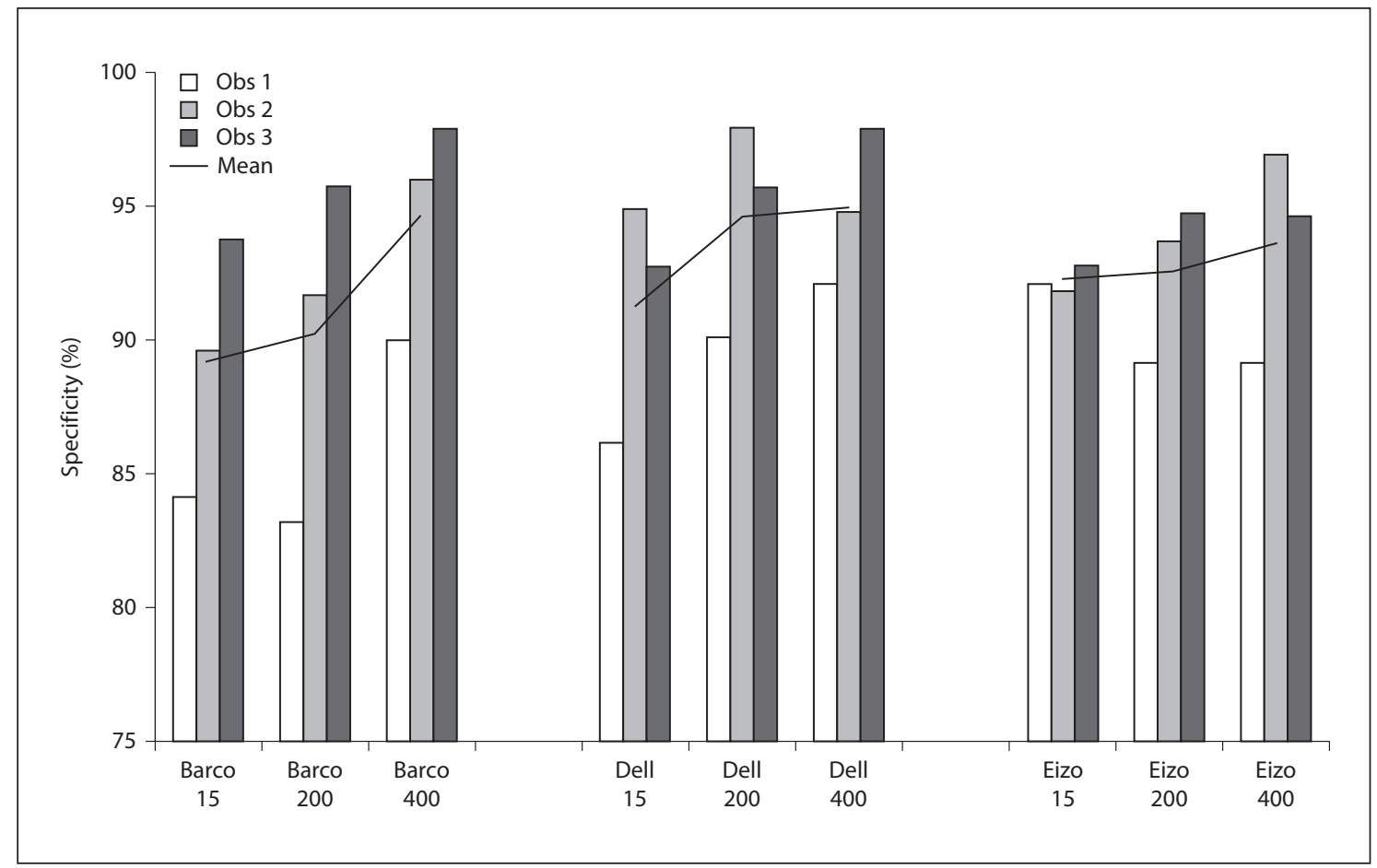

Fig. 2. Specificity for the 3 observers and the mean value (black line) for the 3 room illuminance levels (lux on the display surface) and displays. 


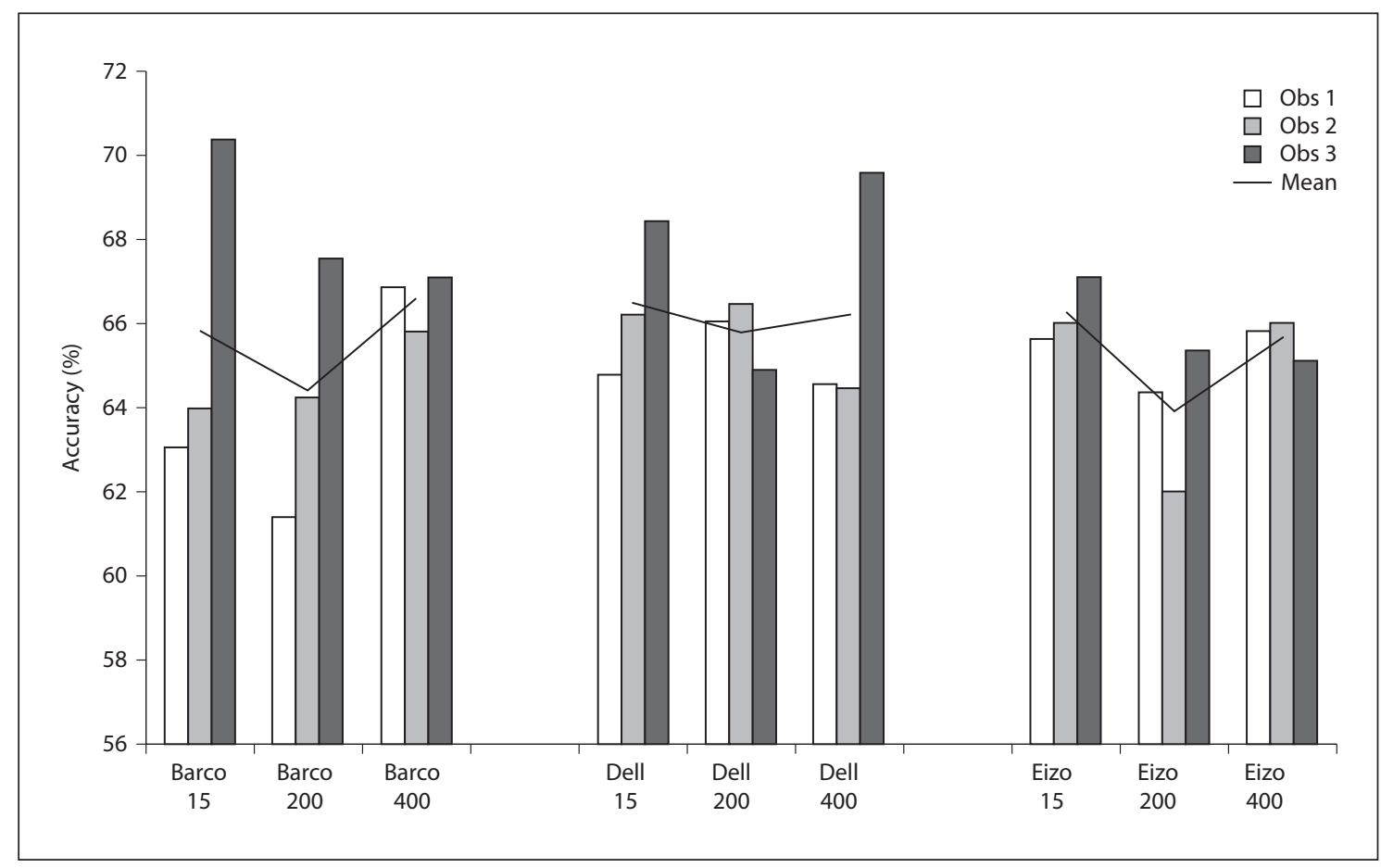

Fig. 3. Overall accuracy for the 3 observers and the mean value for the illuminance levels (lux on the display surface) and displays.

provide an indication of the patient's overall carious challenge. Early radiographic detection of small initial carious lesions enables the timely and effective administration of prophylactic measures. The study was designed to replicate the clinical setting, where non-cavitated carious lesions restricted to the enamel often will be radiographically diagnosed in suboptimal lighting conditions.

Three observers participated in the study. It has previously been demonstrated that the statistical power of a study on accuracy in caries detection is determined by the total number of observations made rather than the number of observers [Hintze et al., 2003]. For our data and defined lesion depth scale (binned 0-3), considering also the amount of observed images, monitors and luminance levels, it was considered sufficient to have 3 observers for the evaluation task. They performed rather differently with relatively high inter-observer variations. These results are similar to previous studies [Hellén-Halme et al., 2008]. The scores for sensitivity were somewhat low, also similar to earlier studies [Haiter-Neto et al., 2009; Bottenberg et al., 2011] and are most likely due to the fact that the lesions included in the sample were mostly small and confined to the enamel.

Effect of Displays and Room Lighting on Caries Diagnostics
In earlier studies, lower illuminance levels were found to significantly improve diagnostic accuracy in the detection of caries lesions [Hellén-Halme et al., 2009] and wrist fractures [Brennan et al., 2007]. Consequently, recommendations have been issued [Samei et al., 2005; STUK, 2008] advocating appropriately low illuminance levels while evaluating radiographs. Our data also indicate a statistically significant advantage in sensitivity at lower (15 lux) illuminance levels compared to the higher levels appropriate for clinical dental work. However, our data also show a comparable decrease in specificity at lower illuminance levels. The higher specificity scores in higher room illuminance settings, while surprising, might partially be explained by the type of images observed. In optimal viewing conditions, more of the smaller lesions could be identified, but at the risk of some additional false-positive scores. This leads us to stress the importance of the differences in sensitivity rather than specificity or overall correctness in these results and explains the seeming contradiction with earlier results.

In addition to clinical examination, radiography is an important tool in caries diagnostics. Most dental radiography is currently done in digital form, which requires the 
optimization of several technical stages from image acquisition to viewing conditions. The final stage in this process is the evaluation of the acquired data on a computer display at various illuminance levels. A practising dentist typically views dozens of intra-oral radiographs weekly to assist in diagnosing carious lesions. A dentist's clinical work, however, requires a level of room illuminance, which is far in excess of that recommended for diagnostic viewing, and changing the lighting while viewing radiographs at the chair-side is not usually possible or convenient. Our hypothesis was that higher display luminance could compensate for this, but our evidence suggests otherwise. A recent study [Hellén-Halme et al., 2011] has found that the luminance level in LCD monitors deteriorates fairly rapidly at the beginning until the deterioration rate starts to level off after approximately $2,000 \mathrm{~h}$ of use. These results are similar, regardless of the initial luminance values of the displays. Whereas in the studied range of displays our study did not find any significant advantage in diagnostic accuracy with higher-end DICOM calibrated displays, these results would indicate a possibly longer useful life for displays with a higher initial luminance value.

Our results did indicate that sensitivity was better in reduced lighting. The higher illuminance required by most dental clinical work does not prevent adequate secondary viewing of radiographs for caries diagnostics, but to ascertain appropriate sensitivity it would probably be advisable to confirm diagnosis in subdued lighting, as suggested by earlier guidelines. Our hypothesis was that higher display luminance could compensate for this higher room illuminance, but our results suggest this is not the case. This would lead us to conclude that the quality of the display does not seem to have a significant effect on the reliability of caries diagnostics in dental digital radiography.

\section{Acknowledgements}

Observations of carious lesions in radiographs were performed by M. Ekholm, T. Pakkala and E. Pakbaznejad. The data was analyzed and statistically tested by M. Frydenberg (biostatistician) and A. Wenzel.

\section{Disclosure Statement}

None of the authors have any conflicts of interest to declare.

\section{References}

Bottenberg P, Jacquet W, Stachniss V, Wellnitz J, Schulte AG: Detection of cavitated or noncavitated approximal enamel caries lesions using CMOS and CCD digital X-ray sensors and conventional D and F-speed films at different exposure conditions. Am J Dent 2011; 24:74-78.

Brennan PC, McEntee M, Evanoff M, Phillips P, O'Connor WT, Manning DJ: Ambient lighting: effect of illumination on soft-copy viewing of radiographs of the wrist. AJR Am J Roentgenol 2007;188:177-180.

-Haiter-Neto F, Casanova MS, Frydenberg M, Wenzel A: Task-specific enhancement filters in storage phosphor images from the Vistascan system for detection of proximal caries lesions of known size. Oral Surg Oral Med Oral Pathol Oral Radiol Endod 2009;107: $116-121$.

-Hellén-Halme K, Hellén-Halme B, Wenzel A: The effect of aging on luminance of standard liquid crystal display (LCD) monitors. Oral Surg Oral Med Oral Pathol Oral Radiol Endod 2011;112:237-242.
Hellén-Halme K, Nilsson M, Petersson A: Digital radiography in general dental practice. A field study. Dentomaxillofacial Radiol 2007; 36:249-255.

Hellén-Halme K, Nilsson M, Petersson A: Effect of ambient light and monitor brightness and contrast settings on the detection of approximal caries in digital radiographs. An in vitro study. Dentomaxillofacial Radiol 2008; 37:380-384.

Hellén-Halme K, Nilsson M, Petersson A: Effect of monitors on approximal caries detection in digital radiographs - standard versus precalibrated DICOM part 14 displays: an in vitro study. Oral Surg Oral Med Oral Pathol Oral Radiol Endod 2009;107:716-720.

Hintze H, Frydenberg M, Wenzel A: Influence of number of surfaces and observers on statistical power in a multiobserver ROC radiographic caries detection study. Caries Res 2003;37:200-205.

IPEM (Institute of Physics and Engineering in Medicine): Recommended standards for the routine performance testing of diagnostic $\mathrm{X}$ ray imaging systems. IPEM Report No. 91. York, IPEM, 2005.
Isidor S, Faaborg-Andersen M, Hintze $\mathrm{H}$, Kirkevang L-L, Frydenberg M, Haiter-Neto F, Wenzel A: Effect of monitor display on detection of approximal caries lesions in digital radiographs. Dentomaxillofac Radiol 2009; 38:537-541.

NEMA (National Electrical Manufacturers Association): 'Digital Imaging and Communications in Medicine (DICOM). Part 14: Grayscale Standard Display Function'. NEMA PS 3.14-2008, 2008.

-Samei E, Badano A, Chakraborty D, Compton K, Cornelius C, Corrigan K, Flynn MJ, Hemminger B, Hangiandreou N, Johnson J, Moxley-Stevens DM, Pavlicek W, Roehrig H, Rutz L, Shepard J, Uzenoff RA, Wang J, Willis CE: AAPM TG18. Assessment of display performance for medical imaging systems: executive summary of AAPM TG18 report. Med Phys 2005;32:1205-1225.

STUK (Radiation and Nuclear Safety Authority): Tervyedenhuollon röntgenlaitteiden laadunvalvontaopass. STUK TIEDOTTAA 2008;2:61-62. 